\title{
Virtual and Augmented Reality for Disaster Risk Reduction
}

\author{
Massimo Migliorini ${ }^{1}$, Luca Licata ${ }^{2}$, Davide Strumendo ${ }^{3}$
}

\author{
(1) Senior Researcher, Fondazione LINKS, massimo.migliorini@linksfoundation.com \\ (2) Company Manager, LD Multimedia, lucalicata@ldmultimedia.net \\ (3) CEO, Funix, davide@funix.it
}

Keywords: Virtual Reality, Augmented Reality, Disaster Risk, Training, Natural Hazards

\begin{abstract}
During a catastrophic event, the ability of the personnel responsible for responding to such an event is due not only to a pre-existing knowledge or ability, but also to the degree of familiarity with the scenario that must be faced. Especially in the case of high-impact but potentially unlikely events, an appropriate response is based on the fact that staff are able to perform their assigned tasks with precision, and to coordinate efficiently with other operators. Psychological effects of stress due to having to face a situation to which the staff is unfamiliar can have a significant impact on performance, thus leading to a degradation in the effectiveness of the intervention and the consequent loss of human life.
\end{abstract}

The recent development of technologies in the field of virtual reality and augmented reality has made available a new level of interaction between people, generating new ways of communicating and transmitting knowledge, which can strongly improve emergency operators training effectiveness.

The simulated reality environments can offer all the characteristics of conventional training techniques by reducing costs and development times of real exercises. In a virtual reality simulation, players can communicate, move from one point to another in an immersive 3D digital environment, perform manual actions, work on machineries, drive vehicles, interact with objects, events and other players hosted in the environment, everything in real-time. Each character is able to see the other players and their actions and can react by taking other actions, within a digital scenario that reproduces the real environment with a high level of fidelity. The simulations can be controlled by the figure of the "masters" (instructors) who can remotely follow all the actions performed by the operators who are carrying out the virtual simulation, create localized events (such as the breaking of a pipe, or the exhaustion of a material necessary for industrial processes) or introducing new critical issues such as an interruption of the electrical or telephone lines.

Virtual training systems also allow and facilitate the collaborative training of geographically distributed personnel; for example, virtual simulation can provide a consistent and synchronized training platform in the event of natural disasters such as earthquakes and hurricanes that require a geographically distributed response; in addition, a virtual reality system can make it possible to verify the efficiency of an emergency plan, thus identifying gaps and aspects that need to be improved.

It thus emerge a strong need to explore the potential of this evolving technology, creating innovative application case able to demonstrate all it potential in improving emergency operators training 


\section{1- Introduction}

The recent development of technologies in the field of virtual reality and augmented reality has made available a new level of interaction between people, generating new ways of communicating and transmitting knowledge.

The term Virtual Reality (VR) refers to a combination of technologies able to immerse the user in a simulated 3D digital environment, and to generate interaction mechanics through which the user can interact with the environment and with other users.

For example, in a virtual reality simulation, players can communicate, move from one point to another in an immersive 3D digital environment, perform manual actions, work on machineries, drive vehicles, interact with objects, events and other players hosted in the environment, everything in real-time. Each character is able to see the other players and their actions and can react by taking other actions, within a digital scenario that reproduces the real environment with a high level of fidelity. The simulations can be controlled by the figure of the "masters" (simulation governors) who can remotely follow all the actions performed by the operators who are carrying out the virtual simulation, create localized events (such as the breaking of a pipe, or the exhaustion of a material necessary for industrial processes) or introducing new critical issues such as an interruption of the electrical or telephone lines.

The term Augmented Reality (AR) instead refers to a combination of technologies capable of "increasing" reality by generating digital content that the user perceives as an integral part of their perspective, superimposed on real reality.

Augmented reality can be useful for example to know more details about an object, identify a path, design systems in a certain context of spaces and furnishings, view the profile of a distant person as if in the same environment in which the user is.

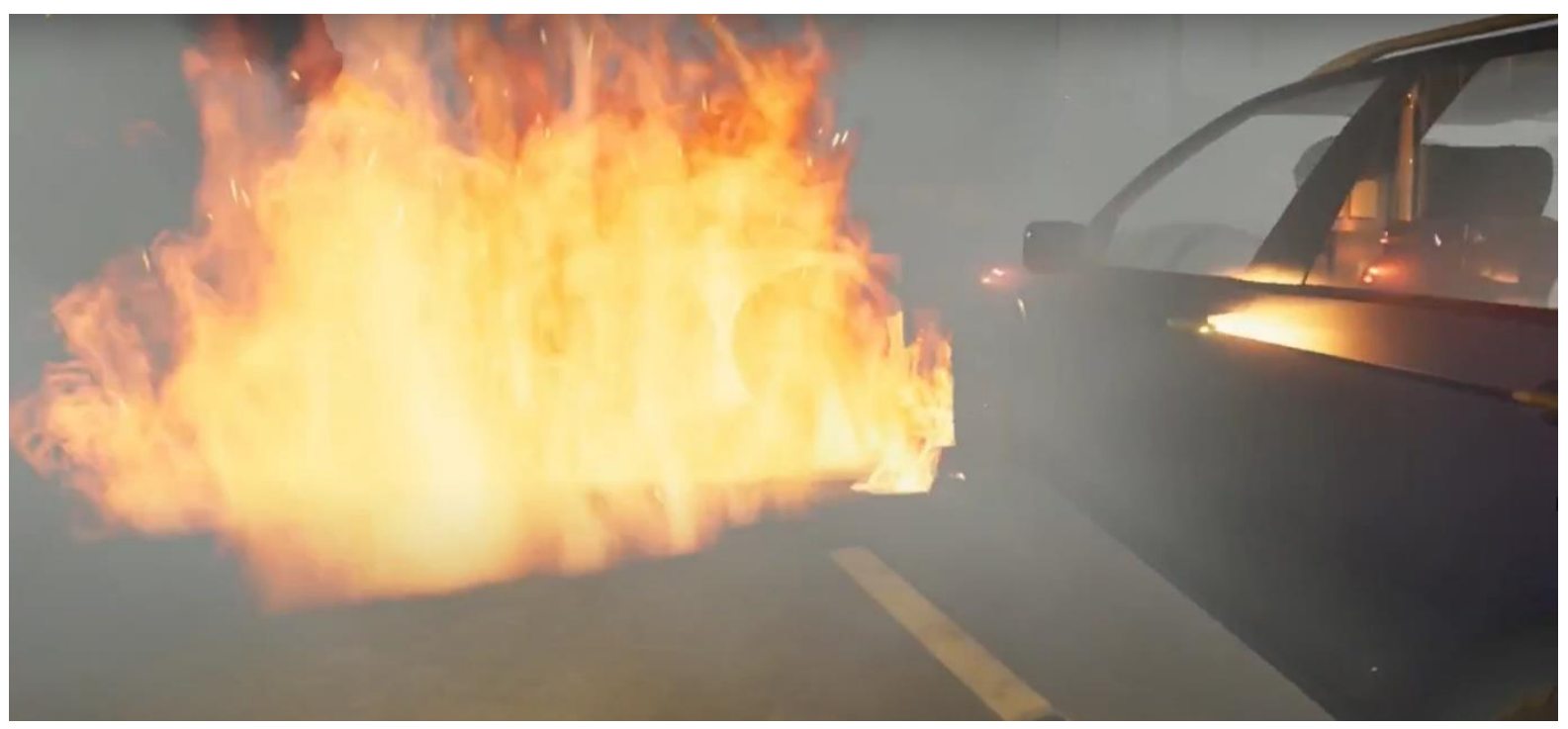

Figure 1. Virtual Reality Simulation - Car accident with fire development

During a catastrophic event, the ability of the personnel responsible for responding to such an event is due not only to a pre-existing knowledge or ability, but also to the degree of familiarity with the scenario that must be faced. Especially in the case of high-impact but potentially unlikely events, an appropriate response is based on the fact that staff are able to perform their assigned tasks with precision, and to coordinate efficiently with other operators. Psychological effects of stress due to having to face a situation to which the staff is unfamiliar can have a significant impact on performance, thus leading to a degradation in the effectiveness of the intervention and the consequent loss of human life.

Efficient training is a key point in preparing for emergency management. The quality, consistency and frequency of training are key elements in the preparation of operators. On the other hand, if the importance of training is clearly recognized, there are factors that hinder its diffusion: the time required, 
costs and safety limits. These factors severely limit the possibility of adequately training "public safety" operators, and this is particularly evident in cases of large-scale mobilization where the preparation of operators can heavily affect the quality of the intervention.

Furthermore, the training scenarios for disasters and natural disasters, in order to be realistic and adequately represent the dynamics of an emergency situation, should incorporate a series of "critical" elements, such as:

- the presence of numerous victims and trapped civilians, often in situations of panic;

- the presence of damage to structures, which progressively evolve, or to assets (eg works of art);

- the presence of fumes and toxic substances;

- the presence of visual and auditory impediments for operators;

- the possibility to train even in contexts that are not normally accessible (eg areas of historical, cultural or environmental value) and in times when there may be difficulties (eg in winter the firefighters may encounter greater difficulties in exercising with the hydrants as the water is subject to freezing);

- the certainty of not interfering with the normal functionality of the structures (e.g. closure of traffic to practice in a tunnel);

- the certainty of a weather suitable for the risk situations represented (eg wind, clouds, etc.).

Including these elements in the training scenario can be costly, dangerous, and in some cases impossible.

A virtual reality scenario, on the other hand, can approximate these elements, maintaining all the advantages of a controlled simulation environment. In this way it is possible to train staff to respond to particularly stressful real conditions, thus improving the ability to make decisions in critical conditions.

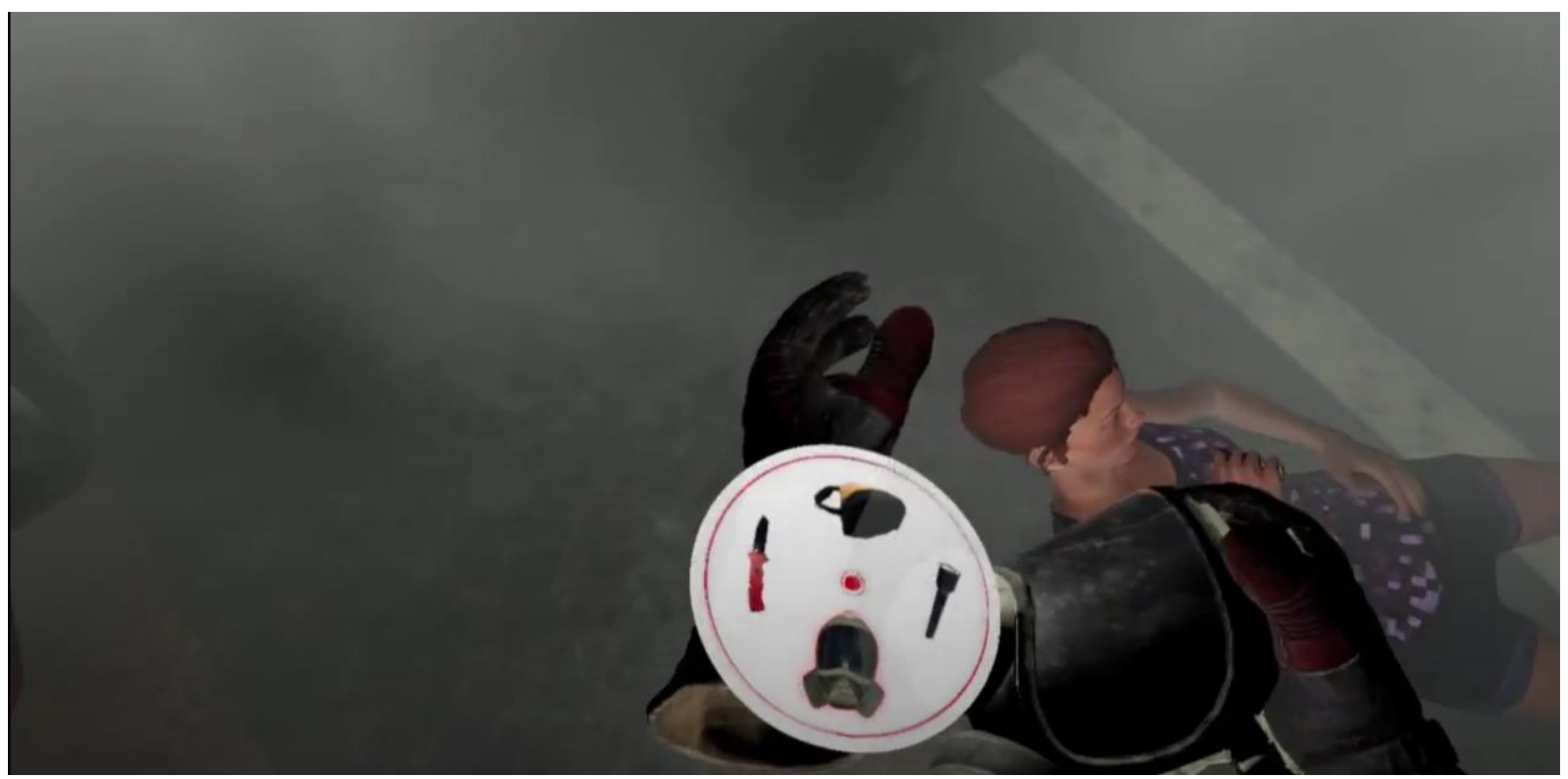

Figure 2. Virtual Reality Simulation - First responders saving a intoxicated victim

The recent improvement of virtual reality technologies has made it possible to represent in an increasingly realistic way the situations for which you want to train the staff, thus becoming an alternative form of training to the conventional one.The simulated virtual reality environments can therefore offer all the characteristics of conventional training techniques by reducing costs and development times of real exercises. Training based on virtual reality technologies for the simulation of catastrophic events can be sized according to the specific use and the organization that decides to adopt virtual reality for training. For example, virtual scenarios can be developed to provide the user with instant feedback on the inputs received (as in the case of the organization of triage for the 
management of natural disasters); in addition, a virtual reality system can make it possible to verify the efficiency of an emergency plan, thus identifying gaps and aspects that need to be improved.

Virtual training systems allow and facilitate the collaborative training of geographically distributed personnel; for example, virtual simulation can provide a consistent and synchronized training platform in the event of natural disasters such as earthquakes and hurricanes that require a geographically distributed response.

Traditional teaching methods such as slide presentations can be easily incorporated into virtual simulation systems, thus making them accessible in a new form. While the benefits that a virtual simulation system can convey to the user in terms of visual stimuli are evident, it is also important to underline the advantage in terms of acoustic stimuli that can be inserted into virtual environments.

\section{2- Benefits Vs Limits}

Compared to traditional training, virtual reality-based training systems therefore have a number of advantages. From an environmental point of view, it is easy to present realistic life scenarios that incorporate other users; in addition, it is possible to manage user inputs to provide feedback in real time. By populating the simulated avatar environments (representation of users in the virtual world) it is possible to provide the trainee with immediate feedback. Behavior modules can be assigned to various avatars to manage the decisions made by the personnel being trained.

Virtual reality systems also allow you to calibrate the level of difficulty according to the degree of preparation of those who must be trained. During the training it is possible to interact both with objects that make up the virtual environment and with other participants in the training; the interaction between participants (real and virtual) can take place through text and / or voice communication, also allowing the simulation of different cultural and socio-economic scenarios. The virtual simulation can be frozen (paused) allowing instructors to discuss with the trainee how best to deal with unexpected events. If necessary, the exercise can be repeated, introducing a series of variations controlled by the instructor. Environmental features can be easily incorporated: buildings, vegetation, humans and sounds are just some of the elements that can be incorporated. Virtual scenarios such as the creation of safety zones within urban areas with a high population density can be easily created, in spite of real large-scale exercises that would often require too much time and cost. These latter aspects are among the major benefits of virtual training systems: real exercises can require costs that are beyond the reach of most administrations. Virtual reality offers a valid alternative by incorporating adequate realism at a fraction of the costs that real exercises would have. Furthermore, the flexibility of the virtual scenario allows to simulate different conditions, thus allowing to evaluate the possible results in the face of different simulation conditions.

Finally, the digital nature of virtual simulations can allow data to be archived for later study and evaluation. This can be an additional added value in the case of emergency management, as it allows us to better understand behavioral phenomena and therefore to prepare improved training procedures. It is important to remember that virtual reality must be seen as a complement to traditional training methods and not as a pure substitute.

Also, AR / VR technologies are able to significantly increase the efficiency of communication and training processes, in particular through the following mechanisms:

- more effective learning compared to normal methods ("living" the situations people remember the notions more easily);

- reduction of costs, since the use of digital content does not require the use of materials, the relocation of people and / or vehicles, and in the case of complex training processes (eg exercises for emergency operators) greater degree of replicability (even two-three times a day);

- applicability to any sector and context: digital scenarios can reproduce any type of environment, objects, effects, etc. and be calibrated on the basis of the specific needs of the context, the type of training and / or learning attitudes of the users, with the possibility of 
inserting elements of difficulty in real time to test the reaction times of the candidates with respect to sudden situations;

- elimination of geographical barriers: thanks to the possibility of using AR / VR contents remotely, users can interact even from geographically distant places, experiencing the same situation together and interacting with it. This aspect generates a very high series of possibilities, for example Italian civil protection operators could conduct daily exercises and experiment with new intervention protocols with the civil protection of any other state;

- simultaneous interconnection of many users: AR / VR technology combined with the internet makes it possible to create multi-player scenarios where multiple users interact with each other in an immersive digital environment. This is an element capable of generating new logics for the planning of training processes (training "on the field" in a digital environment, the possibility of training several operators at the same time and strengthening the coordination mechanics of the teams);

- automatic evaluation of "errors": having the ability to record movements in space, each user can be constantly tracked in every action. This makes it possible to create automatic error evaluation algorithms, giving indications to constantly improve a given physical action or procedure, both individually and in a team.

On the other hand, those technologies still present significant limits. Example of these limits are:

- Problems of motion sickness (vertigo), which depends of graphic quality, speed of moving the frame, imposed movement mechanisms in the digital environment (shifting, armswing, legswing, free movement, etc.)

- Inability to reproduce other senses (heat, smell, etc.) that are part of the reproduced scenario.

We must always remember that a virtual environment does not replace a real scenario in its entirety: it does only in a percentage, around 30-40\% (environments, actions, coordination between people). However, a virtual exercise can be repeated many times, at very low cost. The operators therefore learn perfectly how to move on some difficulty components of the challenge. When the real exercise is then carried out, the operators can work directly on the new elements of difficulty (heat, weight, smells, etc.) starting from a full mastery of all the other elements.

\section{3- Research opportunities}

AR / VR technologies, whose development is constantly growing, open up an infinite series of potential applications.

Example of possible lines focusing on DRR Sector and in particular on the Training of emergency operators, are:

- VR: Design of new modes of movement in a digital environment;

- VR: Design of interactive virtual tours ("game book" style) for training purposes;

- AR: design of AR content to support physical and digital interactions capable of guiding onthe-job learning of manual procedures (eg hooking up a hose, choosing the right tool, etc.);

- VR / AR: Definition of innovative gamification algorithms (connection with SAFE network)

- VR / AR: Design of new tools and systems for representing the most recurring needs in a digital environment (tracking of companions, dialogues, writing, reporting, geo-referencing, management of objects and inventories, etc.);

- VR / AR Design of technological platforms to support the exercises, and refinement of training protocols to allow you to maximize the efficiency of the VR and AR components;

- VR / AR: Design of innovative algorithms for the evaluation of candidates (scores, calculation, reporting). 


\section{4- Conclusions}

The recent development of technologies in the field of virtual reality and augmented reality has made available a new level of interaction between people, generating new ways of communicating and transmitting knowledge, which can strongly improve emergency operators training effectiveness.

Virtual and Augmented reality technologies have demonstrate to have a number of strategic advantages when they are used to train operators, including more effective learning, reduction of costs, applicability to any sector and context, elimination of geographical barriers, simultaneous interconnection of many user, automatic evaluation of "errors". As those technologies are constantly evolving and changing, the development of research initiatives aimed at identifying, evaluating, initiating and carrying out their potential appears to be of high strategic importance, capable of generating strong innovation on the territory. 


\section{Acknowledgements}

A special thanks to Prodige Project staff, LD Multimedia and Funix Company.

\section{References}

[1] M. Migliorini, A. Filieri, F. Moretti, V. Dolci, L. Licata, F. Lamberti, A. Sanna, L. Zigiotti, F. Dellanoce, G. Todesco, P. Sansa, J.C Julien, L. Ansel, S. Fogliacco, D. Galliano, D. Calandra, M. Billi (2017) "Progetto PRODIGE - Proteggere i cittadini, difendere le infrastrutture, gestire i grandi eventi”, retrievable at: http://www.pro-prodige.eu/fileadmin/prodige/contents/download/PUBBLICAZIONE_PRODIGE_ITA.pdf

[2] C.A. Franzon (2016) VR Magazine, “ Il primo intervento chirurgico a 360”, retrievable at: http://realtavirtuale.net/altre-realta/il-primo-intervento-chirurgico-in-live-a-360-gradi/

[3] R. Di Giulio et al (2016) INCEPTION Project, retrievable at: https://www.inception-project.eu/en

[4] M. Ioannides et al, (2018), VIMM+ Project, retrievable at: http://www.vi-mm.eu/

[5] J. Martins et al (2017), Cost Action TD 1406, retrievable at: https://www.cost.eu/actions/TD1406/\#tabs|Name:overview

[6] Massimo Migliorini, JennySjåstad Hagen, Jadranka Mihaljević, Jaroslav Mysiak, Jean-Louis Rossi, Alexander Siegmund, Khachatur Meliksetian, Debarati Guha Sapir (2019) Data interoperability for disaster risk reduction in Europe, Disaster Prevention and Management Journal, ISSN: 0965-3562, https://www.emerald.com/insight/content/doi/10.1108/DPM-09-2019-0291/full/html

[7] D. Calandra, F. Lamberti and M. Migliorini, "On the Usability of Consumer Locomotion Techniques in Serious Games: Comparing Arm Swinging, Treadmills and Walk-in-Place," 2019 IEEE 9th International Conference on Consumer Electronics (ICCE-Berlin), Berlin, Germany, 2019, pp. 348-352, doi: 10.1109/ICCEBerlin47944.2019.8966165. Retrievable at https://ieexplore.ieee.org/abstract/document/8966165 